\title{
Condiciones para la docencia inclusiva: análisis desde las barreras y los facilitadores
}

Judith Pérez-Castro

\section{RESUMEN}

El objetivo de este artículo es analizar las barreras y facilitadores identificados por una muestra de estudiantes con discapacidad, de dos universidades públicas mexicanas, en tres componentes de la docencia: las formas de presentación, las formas de participación y compromiso, y las actitudes hacia la inclusión. La investigación consistió en un estudio de caso y la técnica de indagación fue la entrevista a profundidad. Los resultados nos muestran que la formación docente en inclusión y discapacidad es importante, pero, que se requiere brindar herramientas que permitan a los profesores hacer ajustes razonables, con base en las necesidades de los estudiantes.

Palabras clave: docencia, barreras, facilitadores, discapacidad, educación superior, México. 


\title{
Condições para a docência inclusiva: análise a partir das barreiras e os facilitadores
}

\section{RESUMO}

O objetivo deste artigo é analisar as barreiras e facilitadores identificados por uma amostra de estudantes com deficiência, de duas universidades públicas mexicanas, em três componentes da docência: as formas de apresentação, as formas de participação e compromisso, e as atitudes para a inclusão. A pesquisa consistiu em um estudo de caso e a técnica de indagação foi a entrevista a profundidade. Os resultados mostram que a formação docente em inclusão e deficiência é importante, mas, também, se requer brindar ferramentas que permitam aos professores fazer ajustes razoáveis, com base nas necessidades dos estudantes.

Palavras chave: docência, barreiras, facilitadores, deficiência, educação superior, México.

\section{Conditions for inclusive teaching: analysis from barriers and facilitators}

\section{ABSTRACT}

The objective of this article is to analyze the barriers and facilitators identified by a sample of students with disabilities enrolled in two Mexican public universities, in three components of teaching: the ways in which they are presented, the ways in which they are invited to take part in the activities and their level of commitment and the attitudes towards inclusion. The research consisted of a case study and the investigacion technique was the in-depth interview. The results show us that teacher training in inclusion and disability is important, but it is also necessary to provide tools that allow teachers to make reasonable adjustments, based on the needs of the students.
\end{abstract}

Key words: teaching, barriers, facilitators, disability, higher education, Mexico. 


\section{Introducción}

La docencia es una de las funciones sustantivas de las instituciones de educación superior (IES). Su estatus ha sido ampliamente discutido, pasando de aquellos que la consideran "como una actividad artística (que depende de las cualidades del artista y sólo depende su criterio)", a los que la conciben como una "actividad científica (lo que significa entenderla como sujeta a las normas que imponen las evidencias acumuladas)" (Zabalza, 2009: 71). En lo que sí hay coincidencia es en que se trata de una labor compleja, que va más allá del uso de determinadas técnicas para trasmitir conocimiento, sino que requiere de la formación continua y el compromiso decidido de quienes la ejercen (Imbernón, 2000; Jackson, 2001; Zabalza et al., 2012).

En el nivel superior, la docencia está articulada con el desarrollo de los campos disciplinarios y el cumplimiento de otras funciones, como la investigación y la difusión del conocimiento. Más recientemente, se le ha vinculado con otras tareas, como las tutorías, la construcción de plataformas de aprendizaje innovadoras, el aprovechamiento de las nuevas tecnologías y el seguimiento de los impactos de la enseñanza, entre otros (Hénard y Roseveare, 2012). En esta diversidad de demandas, se inscribe el tema de la inclusión de las personas con discapacidad.

Impulsado por las políticas compensatorias de los años ochenta del siglo pasado, el acceso de las personas con discapacidad, y de otros colectivos en situación de 'vulnerabilidad' o 'riesgo social', fue abriéndose paso en el discurso y las acciones educativas (Bolívar, 2012). El enfoque inicial fue de tipo integracionista, pero, hacia mediados de los noventa, los sistemas educativos de algunos países desarrollados, iniciaron el camino de lo que posteriormente se conocería como las reformas inclusivas (Parrilla, 2002). A partir del siglo XXI, "a nivel internacional, se aboga por una 'escuela inclusiva' que maximice las oportunidades de éxito de cada individuo o grupo [...] Se multiplican los programas y los dispositivos, así como los objetivos públicos a los que se dirigen" (Bolívar, 2012: 35).

La población con discapacidad empezó a formar parte de la demanda en la educación superior hacia finales de la década de los noventa, en países como Gran Bretaña, Estados Unidos, Noruega y España (OECD, 2003), mientras que en América Latina logra tener mayor presencia hasta el 2000 (Moreno, 2005). Esto supuso una serie de desafíos para las instituciones y en particular para sus académicos, dado que son ellos los que asumen la tarea de trabajar con estudiantes cada vez más diversos.

En una extensa revisión de la producción reciente, Lawrie et al. (2017) encontraron que la inclusión en la enseñanza y el aprendizaje tiende a abordarse desde las estrategias pedagógicas. Más aún, prevalecen los trabajos que ven a las condiciones del aula de manera aislada, con poca o nula relación con el contexto institucional. Las principales conclusiones a las que llegaron estos autores fueron las siguientes:

1. Hay una diversidad de formas de entender la inclusión y sus implicaciones. En ocasiones, el interés se ha centrado en las estrategias pedagógicas para responder a las necesidades de los estudiantes y la eliminación de las barreras, mientras que en otras la atención se ha puesto en la accesibilidad y la colaboración de las partes interesadas (stakeholders). También, se le ha considerado como un proceso de equilibración entre los estándares académicos y la evaluación multimodal y, finalmente, se le ha definido como un enfoque holístico-comprehensivo para la enseñanza y el aprendizaje de diferentes colectivos de estudiantes.

2. El Diseño Universal para el Aprendizaje (DUA) constituye una importante base para el logro de un currículum inclusivo, no sólo porque beneficia a los aprendizajes y el compromiso de los alumnos, sino porque promueve el trabajo colaborativo entre ellos y los profesores; además 
contribuye al desarrollo profesional, recupera la experiencia docente y la formación disciplinaria previa, e involucra la dimensión institucional. Diseñar desde este enfoque siempre será más pertinente que implementar ajustes especiales o remediales al currículum, que sólo mitigan las barreras o incluso pueden crear nuevas.

3. La presentación inclusiva de los contenidos curriculares conlleva la combinación de estrategias formales e informales de aprendizaje, así como el uso de múltiples formas de exposición de los contenidos y medios para la participación. Todos los alumnos, con o sin discapacidad, afrontan barreras en este aspecto, de ahí la importancia de reconocer la multidimensionalidad del trabajo colaborativo y comprender los factores que intervienen en el funcionamiento del grupo, así como las vías para que los estudiantes se sientan incluidos en el proceso de aprendizaje y respetados como personas.

4. La evaluación inclusiva plantea múltiples retos a los docentes, porque las estrategias más utilizadas por los profesores y las instituciones suelen producir barreras, en especial para los estudiantes en situación de desventaja, o por la dificultad para conciliar el enfoque de la inclusión con altos estándares académicos. Reconocer estos problemas supone desarrollar formas alternativas y flexibles de evaluación, sustentadas en procesos formativos tempranos y en la realimentación.

5. En la enseñanza y el aprendizaje inclusivos no sólo intervienen las condiciones de la clase, sino también las institucionales. La creación de una cultura de la inclusión, la combinación de estrategias de tipo descendente (top-down) y ascendente (bottom-up), la coordinación de apoyos y servicios de atención a nivel central, el desarrollo de herramientas para analizar los factores que intervienen en la disociación entre los miembros de la comunidad y las instituciones, son algunas de las medidas que abonan al compromiso institucional con el aprendizaje y la enseñanza inclusivos.

El objetivo de este artículo es discutir tres elementos de la docencia inclusiva en el nivel superior: las formas de presentación, las formas de participación y compromiso, y las actitudes hacia la inclusión. Los resultados se desprenden de una investigación realizada en dos universidades públicas mexicanas, financiada por el Programa de Apoyo a Proyectos de Investigación e Innovación Tecnológica (PAPITT), de la Universidad Nacional Autónoma de México (UNAM). El texto está organizado en cinco apartados: en el primero abordamos las barreras y facilitadores para el desarrollo de prácticas docentes inclusivas, a partir de los tres componentes anteriormente señalados; en el segundo exponemos la ruta metodológica que siguió la investigación; el tercer y cuarto apartados incluyen los testimonios de los estudiantes con discapacidad sobre las acciones implementadas por sus profesores y el análisis de resultados; finalmente, hacemos algunas consideraciones sobre lo aquí expuesto.

\section{Barreras y facilitadores para la docencia inclusiva}

Diversos autores han señalado que la inclusión es un proceso dilemático en muchos sentidos. En particular, para el profesorado, la disyuntiva está en atender a cada estudiante y al mismo tiempo trabajar colectivamente (Ainscow et al., 2001), responder a las diferencias sin generar o reforzar los estigmas (Florian $e t$ al., 2011), desarrollar ambientes inclusivos y simultáneamente lidiar con otras exigencias, como la rendición de cuentas, la maximización de los recursos y la imagen pública sobre su desempeño (McKay, 2016). En suma, como advierte Norwich (2008), el dilema básico es reconocer o no las diferencias, a pesar de los riesgos en términos de la estigmatización o menoscabo de las personas y la disminución de oportunidades significativas y de calidad. 
Por otra parte, es importante entender que la inclusión no sólo depende de la voluntad de los profesores y estudiantes, ya que ambos enfrentan múltiples barreras, como resultado de factores estructurales, ambientales o actitudinales (French, 2017). Éstas impiden la inclusión plena de las personas con discapacidad y están presentes en todos los niveles educativos. Específicamente, en las barreras para la docencia, confluyen variables tanto de tipo sistémico, como escolares (OMS, 2011). Las primeras tienen que ver con la legislación educativa, la organización de los sistemas de enseñanza, las políticas y programas en materia de discapacidad e inclusión y los recursos financieros; las segundas comprenden los currículos y métodos de enseñanza rígidos, la poca formación de los profesores, las barreras físicas y actitudinales, así como los actos de violencia, intimidación o abuso hacia estas personas (OMS, 2011).

Igualmente, diversos trabajos coinciden en que los profesores pueden ser al mismo tiempo agentes facilitadores (Ainscow, 1995; Hewett et al., 2017) o limitantes para la inclusión de los estudiantes con discapacidad (Delgado-Sanoja et al., 2016; Florian et al., 2010; Moriña y Carballo, 2018). Pero en este proceso hay otras cosas en juego, algunas atañen directamente al docente y otras rebasan su competencia. Para los fines de este artículo, nos interesa tratar las barreras y facilitadores en tres ámbitos de la docencia: las formas de presentación, las formas de participación y compromiso, que son dos de los principios del DUA, y las actitudes hacia la inclusión.

\section{Las formas de presentación}

Éstas comprenden la diversidad de estrategias y recursos utilizados por los profesores para trabajar los contenidos curriculares (Konur, 2006). Aquí, las barreras se presentan cuando las clases se organizan únicamente en torno a la actividad docente, en lugar de centrarse en los aprendizajes de los estudiantes. Uno de los problemas más frecuentes es la toma de notas, en especial, cuando se usa el pizarrón de manera intensiva, se muestran imágenes impresas o diapositivas, o cuando el profesor habla sin hacer pausas; esto pone a los alumnos en la situación de escuchar, tratar de escribir y al mismo tiempo seguir el ritmo de la clase (Fuller et al., 2004). La distribución de lecturas adicionales o notas de clase ampliadas puede servir como facilitadores, pero, si no se consideran las necesidades de los estudiantes, como, el tamaño de letra, el contraste y el espaciado, se convierten en una dificultad adicional (Hopkins, 2011).

Otras barreras resultan del contenido de los cursos, usualmente diseñados para alumnos 'promedio', con una variedad de temas y actividades que no siempre pueden ser completados por todos. Los planes de estudio no suelen ofrecer pautas para hacer ajustes, lo que disminuye las oportunidades de aprendizaje y puede convertirse en una presión adicional. No obstante, los profesores pueden contribuir desde lo que les toca hacer, por ejemplo, Hopkins (2011) propone que, en la descripción de los cursos o módulos, éstos expliquen las estrategias para incluir a los estudiantes con discapacidad, ya sea en los contenidos, el desarrollo de las clases y la evaluación. Otros facilitadores son la implementación de estrategias para el aprendizaje práctico, el uso de tablas y cuadros, la elaboración de notas estructuradas, la enseñanza sistemática a partir de contextos específicos y el aprendizaje para la toma de notas (Scruggs, 2012, citado por Yuknis y Bernstein, 2017).

Las formas de presentación incluyen el acceso a la evaluación, que es un punto crítico para profesores y estudiantes (Konur, 2006). En este tipo de barreras, intervienen distintos factores, algunos externos al docente, como la presencia de distractores, y otros relacionados con él, como la utilización de estrategias tradicionales para la evaluación, el lenguaje y tiempo destinado para contestar los exámenes (Brandt, 2011).

Éste es un asunto complejo porque en él se combinan variables pedagógicas y organizativo-institucionales. Las primeras se refieren a las estrategias y los criterios que utiliza cada profesor, lo que también 
está en función de su perfil profesional y su formación docente; las segundas aluden al régimen académico de cada institución (Baquero et al., 2009), el cual, entre otras cosas, comprende las formas y tiempos para la evaluación, y la escala de calificaciones. Superar estas barreras depende tanto de las prácticas y la sensibilidad de los docentes, como de la cultura y las políticas institucionales con respecto a la discapacidad y la inclusión.

\section{Las formas de participación}

El segundo elemento de la docencia inclusiva que nos interesa discutir es el de las formas de participación, que se remite al compromiso que los alumnos establecen con la clase, así como la manera en que son motivados - por sus profesores y compañerospara hacerlo (AHEAD, 2017). Éstas tienen una estrecha relación con las formas de presentación, ya que las actividades que se planeen y el aprovechamiento que se haga de los espacios y tiempos de la clase pueden favorecerla u obstaculizarla.

$\mathrm{Al}$ respecto, Fuller et al. (2004) han encontrado que las clases expositivas y las sesiones tipo seminario tienden a limitar las posibilidades de que los estudiantes expresen sus ideas o planteen sus dudas, en especial para aquellos con discapacidad auditiva, visual y múltiple, porque tienen que hacer un mayor esfuerzo para escuchar y mantener la interacción con el docente y sus compañeros. Además, los alumnos tienen más dificultades para seguir el ritmo de la clase y encontrar momentos adecuados para participar, principalmente, en las sesiones de preguntas y respuestas o de discusión colectiva (Fuller et al., 2004).

Estos autores han reportado que entre las barreras más frecuentes están: el tiempo asignado para completar las tareas, la dificultad para tomar notas, problemas con las habilidades de lecto-escritura y el tiempo destinado para la lectura de los materiales (Healy et al., 2006). Lo interesante de esta investigación es que también abarcó a una muestra de alumnos sin discapacidad y los resultados apuntaron hacia los mismos problemas, aunque con porcentajes más bajos (Healy et al., 2006), lo que nos recuerda que las prácticas inclusivas benefician a los aprendizajes y a la participación de todos.

Organizar grupos heterogéneos, promover la solución colaborativa de los problemas y diseñar actividades con distintos niveles de complejidad para desarrollar ciertas competencias, son acciones que los profesores pueden implementar para alentar la participación (Fernández, 2013). Otra vía es recurrir a métodos de aprendizaje inclusivos, como la instrucción entre pares, el aula invertida y el aprendizaje combinado, los cuales permiten que los alumnos trabajen con base en sus fortalezas y ritmos, estimulan el trabajo por equipo y fortalecen la autonomía personal (AHEAD, 2017).

\section{Las actitudes para la inclusión}

Las actitudes hacia la discapacidad y la inclusión no sólo dependen de la buena voluntad de las personas, sino también del conocimiento que se tenga al respecto. Especialmente en las instituciones educativas, es importante que los docentes se hagan de herramientas disciplinarias y pedagógicas que les permitan trabajar con grupos cada vez más heterogéneos, por cuestiones de género, ingresos económicos, capacidad, origen étnico o ruralidad (Gairín y Suárez, 2014).

En particular, sobre los alumnos con discapacidad, en un estudio hecho por la organización Red2Red (2013), se encontró que las barreras actitudinales más frecuentes por parte del profesorado eran las bajas expectativas sobre sus conocimientos y habilidades académicas, la falta de comunicación entre ellos y los estudiantes, y la resistencia para realizar ajustes a sus seminarios, aunque estuvieran sancionadas en la normativa institucional.

Otras barreras son la falta de una planeación incluyente de las clases, el desconocimiento de los derechos de los alumnos y en general de la legislación sobre la 
población con discapacidad, y la escasa comunicación entre los profesores y los servicios de atención a los estudiantes (Wilson et al., 2000). De igual forma, se han señalado actos de violencia verbal, como la asignación de sobrenombres, y psicológica, como la intimidación y el aislamiento (OMS, 2011).

Por supuesto, estas barreras no sólo están presentes entre los profesores, sino que han sido reportadas en otros miembros de la comunidad, alumnos, directivos y personal administrativo (Wray, 2011). En el caso de los docentes, superarlas requiere de su disposición y empatía, pero, a la par, necesitan "saber qué hacer ante determinadas situaciones para no tener que actuar de manera improvisada" (Moriña y Carballo, 2018: 90), en temas como la normativa universitaria, los ajustes al currículo y a las actividades áulicas, la elaboración de materiales accesibles, la asignación de los trabajos finales y los formatos de exámenes (Lombardi et al., 2017; Moriña y Carballo, 2018).

Finalmente, todo esto necesita de un buen manejo de la información institucional (OMS, 2011), los profesores deben conocer los programas y apoyos universitarios, disponer de canales de comunicación efectivos con las coordinaciones de los programas, el claustro de profesores y las oficinas de atención a la población estudiantil, así como saber con antelación sobre la presencia de una persona con discapacidad en sus clases.

\section{Metodología}

Los resultados que aquí presentamos se desprenden de una investigación de tipo cualitativo, desarrollada a partir de un estudio de caso intrínseco (Stake, 2003), con el que buscamos recuperar las experiencias de los alumnos con discapacidad, en relación con sus oportunidades educativas y los diferentes aspectos de su formación, es decir, su presencia, participación y aprendizajes en la universidad (Echeita y Ainscow, 2011).

Para delimitar el caso, siguiendo a Stake (2003), establecimos cinco líneas temáticas: 1) Antecedentes sobre la inclusión, que implicó la revisión de las políticas y programas implementados en las últimas dos décadas para la atención a grupos en situación de vulnerabilidad; 2) Condiciones actuales para la inclusión, en donde consideramos desde la normativa institucional hasta la infraestructura; 3) Información estadística sobre los estudiantes con discapacidad; 4) Diversidad de la oferta académica en pregrado y posgrado, así como de los servicios educativos, y 5) Presencia y participación de las personas con discapacidad.

Otros aspectos que tomamos en cuenta fueron: el aporte que los procesos y factores institucionales podían hacer a nuestra comprensión del problema, la factibilidad para realizar las diferentes etapas de la investigación, la relación con los informantes, es decir, con los alumnos con discapacidad, y las facilidades para acceder a las universidades (Rodríguez et al., 1996).

A partir de estas líneas y criterios seleccionamos dos instituciones: la Universidad Nacional Autónoma de México (UNAM) y la Universidad Juárez Autónoma de Tabasco (UJAT).

\section{El contexto de estudio}

La construcción del estudio de caso implicó una amplia exploración de lo que las instituciones seleccionadas estaban haciendo en materia de atención a los estudiantes con discapacidad. Para ello, primeramente analizamos las políticas y programas desarrollados en las dos universidades; a la par, revisamos la información sobre la población estudiantil con discapacidad y, por último, buscamos recuperar otros elementos, documentales o testimoniales, para conocer los alcances de las acciones institucionales y entrar en contacto con los sujetos de estudio. Esto permitió conformar una base con diferentes tipos de datos y referencias, algunos con información muy rica y claramente articulados entre sí, y otros que requirieron un mayor trabajo para su análisis. A continuación, presentamos una síntesis de este proceso. 


\section{El abordaje de la inclusión en la UNAM}

En la UNAM, la atención a la población con discapacidad inició con la provisión de algunos servicios, como el Departamento del Tiflológico para personas con discapacidad visual, inaugurado en 1959, y el deporte en silla de ruedas, en 1972. A partir de los años noventa, algunas entidades de la Universidad empezaron a introducir esta problemática en sus planes de estudio (Ríos et al., 2013), pero fue hasta 2000 que pasó a formar parte de la agenda institucional, de manera más articulada (Brogna y Rosales, 2013).

En 2003 se emitieron los "Lineamientos para dar atención a las personas con capacidades diferentes" - que era la denominación utilizada en ese momento- $y$, diez años después, se creó la Unidad de Atención para Personas con Discapacidad (UNAPDI), en donde se da orientación sobre los servicios institucionales, para que los alumnos puedan incorporarse plenamente a la vida universitaria.

Otras medidas han recaído en el ámbito de la accesibilidad fisica y de información, como el sistema de transporte interno para personas con discapacidad, las adaptaciones arquitectónicas, la Sala Themis en la biblioteca de la Facultad de Derecho y el servicio de consulta especializada en la biblioteca central (Pérez-Castro, 2016). También se dispone del servicio de orientación educativa, la asistencia para el examen de selección, información sobre las becas de manutención y para estudiantes con discapacidad de la Secretaría de Educación Pública (SEP) y el aula-laboratorio de innovación, de la Dirección General de Cómputo y de Tecnologías de Información y Comunicación, entre otros (UNAM-DGACO, 2019).

Con respecto a la legislación, además de la publicación de los lineamientos y la creación de la UNAPDI, la Defensoría de los Derechos Universitarios da asesoría en esta materia a los estudiantes con discapacidad (DDU, 2019) y el Comité de Atención a las Personas con Discapacidad (CADUNAM) brinda orientación sobre los servicios educativos a los que tienen derecho (López, 2015). Otros servicios son el deporte adaptado, las visitas guiadas en algunos museos de la UNAM, así como las instalaciones del Centro Cultural Universitario, la bolsa de trabajo para las personas con discapacidad y la plataforma Descarga Cultura UNAM.

A la fecha, no se dispone de información actualizada sobre la matrícula de los estudiantes con discapacidad, pero se estimaba que, entre 2012 y 2013, había 287 personas con esta condición, entre la licenciatura y el bachillerato (Ríos et al., 2013).

\section{El abordaje de la inclusión en la UJAT}

En la UJAT, el tema de la discapacidad se abordó inicialmente de manera indirecta a través de políticas para el logro de la equidad y la atención a los sectores desfavorecidos (Abdó, 2000). Después, se llevó a cabo la reestructuración del modelo educativo y la reorientación de los programas, como estrategias para alcanzar la equidad en la enseñanza (Gil, 2006). Sin embargo, en ambos casos, la discapacidad estuvo considerada de manera general en las políticas universitarias. Fue hasta 2012 cuando se empezaron a definir algunas líneas de acción para estos estudiantes, las cuales se orientaron fundamentalmente a la adecuación de las instalaciones universitarias (Piña, 2012).

El primer registro que se tiene del ingreso de un estudiante con esta condición fue en 2008, en la carrera de Técnico Superior en Música (Palmeros, 2014). Pero, para el periodo en que realizamos la investigación, había ya 33 alumnos con discapacidad a nivel licenciatura (Piña, 2016).

Las principales medidas que se han implementado a la fecha son la distribución de becas a través del Programa de Apoyo Económico para Estudios de Licenciatura (PAEEL), el programa de asesorías para las tareas y otras actividades académicas a cargo de estudiantes de semestres más avanzados, los asistentes de lectura para el examen de admisión y los cursos de capacitación para la atención de los alumnos con discapacidad, dirigidos a los profesores (Pérez-Castro, 2016). 


\section{Los sujetos de la investigación}

Para acercarnos a los sujetos de estudio, recurrimos a la entrevista a profundidad. Se construyó una muestra intencional, tomando como base dos criterios: abarcar distintos tipos de discapacidad y que los alumnos estuvieran en diferentes momentos de la trayectoria académica.

El guion de entrevista se organizó en siete dimensiones: física, económica, técnica, institucional, social, cultural y natural, que desde el enfoque de vulnerabilidad social, se denominan dimensiones de la interacción humana (Wilches-Chaux, 1993), y se cruzaron con los tres grandes componentes de la inclusión educativa: presencia, participación y aprendizajes (Echeita y Ainscow, 2011).

Antes de hacer la entrevista, se informó a los estudiantes y, cuando fue necesario, a los padres de familia, profesores y autoridades institucionales, sobre los fines de la investigación, garantizando el anonimato, la confidencialidad y el uso responsable de la información. Igualmente, se les explicó el guión y se les dieron las condiciones para reservarse el derecho de detenerla o de no contestar las preguntas que ellos juzgaran convenientes.

Se concretaron 20 entrevistas en total, nueve en la UJAT y 11 en la UNAM. Con base en los dos criterios definidos anteriormente, en la UJAT, la muestra quedó conformada por cinco estudiantes con discapacidad visual, dos con motriz y uno con auditiva. En cuanto a la trayectoria académica, cinco de los entrevistados cursaban los últimos semestres, tres iban a la mitad de la carrera y uno la estaba empezando. En la UNAM, la muestra incluyó a cinco estudiantes con discapacidad motriz, tres con visual y tres con múltiple. Asimismo, tres personas iban a la mitad de la carrera, tres más estaban en los últimos semestres, una había terminado los créditos y cuatro eran recién egresados.

\section{Análisis de la información}

Para analizar la información, primero se transcribieron las entrevistas y después se organizó una matriz de datos en Excel, por institución, siguiendo el orden establecido en el guión. El siguiente paso fue elaborar una segunda matriz, cruzando las categorías de presencia, participación y aprendizaje, con las de barreras y facilitadores. Esto nos permitió identificar algunas temáticas que sobresalían en los testimonios de los estudiantes, como el de las condiciones para el trabajo en el aula con los docentes y con sus compañeros. Finalmente, se compararon los resultados para observar aspectos en común y diferencias entre los entrevistados de las dos universidades.

Por último, cabe señalar que, con el fin de mantener la confidencialidad, elaboramos códigos para referirnos a nuestros sujetos de estudio. De este modo, en E4-F-UJAT la E corresponde a 'estudiante' y 4 al número de la entrevista; $\mathrm{F}$ indica que el género en este caso es femenino, mientras que UJAT alude a la institución de adscripción.

\section{Principales resultados}

De acuerdo con Mckay (2016), las dificultades que enfrentan los profesores que trabajan con grupos diversos, se pueden circunscribir en dos grandes ámbitos: cómo crear ambientes inclusivos y cómo enseñar de manera inclusiva, a lo que se suman las barreras que hemos construido las sociedades discapacitantes.

En los siguientes apartados, presentamos las barreras y facilitadores identificadas por los alumnos con discapacidad, centrándonos en las formas de presentación, de participación y compromiso, y en las actitudes de los docentes. Es pertinente señalar que, aunque se trata de un ejercicio que se basa en los testimonios de sólo uno de los agentes del proceso de enseñanza y aprendizaje, nos parece relevante realizarlo por lo que aporta respecto de los procesos de inclusión (y exclusión) que se dan en las clases. En esta línea se encuentran otros trabajos como los de Aquino et al. (2017), Fuller, Bradley et al. (2004), Vickerman y Blundell (2010), Waterfield et al. (2006) y Wray (2011), en donde a partir de las experiencias de los estudiantes, se plantean líneas de acción para 
la enseñanza, la evaluación de los aprendizajes, el ambiente en el aula y los servicios de atención a la población con discapacidad.

\section{Las barreras y facilitadores para la docencia en la UNAM}

Como hemos dicho, las formas de presentación comprenden las estrategias y recursos en los que se apoya el profesor para comunicar los contenidos del curso. Cada docente, a partir de su campo de conocimiento y formación pedagógico-didáctica, puede tener preferencias o sentirse más cómodo con ciertas modalidades de enseñanza, pero, en cualquier caso, conviene tener en cuenta que se aprende más y mejor cuando el conocimiento se presenta de distintas maneras (AHEAD, 2017).

En este aspecto, las principales barreras señaladas en la UNAM fueron: el predominio de las clases expositivas (cinco estudiantes), el uso de materiales impresos (tres), especialmente para las personas con discapacidad visual, y el tiempo para entregar las tareas (tres).

Era clase magistral, o sea el profesor hablaba y captabas lo que había y, cuando no captabas, lo que había era, te ponían presentaciones o así. Sólo una maestra hace trabajos por equipo y eso, pero fuera de ahí (E20-F-UNAM).

[...] por ejemplo, en materias de estadísticas o matemáticas en las que tenía forzosamente que ver el pizarrón, ésas sí se me dificultaron porque no ligaba con los ejemplos que ponían los maestros en el pizarrón, aunque me sentara mero enfrente, no alcanzaba a ver de todos modos (E11-M-UNAM).

Otras barreras apuntaron hacia los formatos y tiempos asignados para las actividades (cuatro estudiantes) y la realización de prácticas de campo (uno). "Pues, están bien [las clases], pero obviamente no están adaptadas para alguien en estas situaciones, ¿no? Casi siempre tenía que correr para ir como los demás" (E18-M-UNAM).
Entre los facilitadores implementados por los profesores estuvieron: extender los plazos para las tareas y trabajos (tres alumnos), entregarlos vía electrónica (dos) y explicar las clases con más detenimiento (dos).

Sí me lo daban [más tiempo], porque en cierta forma estaban conscientes de que no era tan fácil, en mi caso, hacer los trabajos y, pues sí tenían un poco de conciencia en cuanto a eso (E12-M-UNAM).

$[\ldots]$ con algunos [maestros] es totalmente abierto el asunto, o sea, desde el principio fue, si puedes vienes, si no, nos entregas trabajos, es más, me los mandas por Internet (E19-F-UNAM).

Con respecto a la evaluación, los entrevistados dijeron haber tenido problemas para contestar los exámenes escritos (tres alumnos), cumplir a tiempo con los trabajos finales (tres) y asistir regularmente (dos), cuando era un requisito para acreditar la materia.

Si acaso en los ensayos, cuando eran muy largos, me cansaba [...] Con la computadora, me costaba también un poquito, porque mi mano derecha no puede moverse bien, entonces, coordinar dedos y esas cosas, sí me costaba (E20-F-UNAM).

Algunas materias, no sé, las más pesadas, donde sí importaba mucho que estuviera [presente], como yo tenía que ir varias veces al hospital, pues esas las terminé reprobando (E18-M-UNAM).

En otros casos, el contenido de los cursos dio margen para ajustar la evaluación.

Por ejemplo, en algunas materias, no había como un límite de tiempo en el examen. Podía llevarse dos o tres horas, pero quien iba acabando ya se iba. Entonces, ahí, era cuando podía tomarme más tiempo del que los demás ocupaban y a lo mejor era de las últimas, pero no había restricción de tiempo (E10-F-UNAM). 
Con todo, los entrevistados (cuatro alumnos) sostuvieron que, cuando lo solicitaron, la mayoría de los maestros les extendió el plazo para entregar los trabajos finales.

El segundo aspecto a abordar es el de las formas de participación y compromiso, que están estrechamente vinculadas con las formas de presentación. De acuerdo con los entrevistados, las clases expositivas eran la estrategia más socorrida por sus profesores, lo que les generaba dificultades para mantener el contacto con ellos y sus compañeros (dos alumnos), así como para plantear sus dudas y puntos de vista (tres).

Pues, depende, por ejemplo, en las teóricas, a veces había maestros que llegaban, daban su clase y se iban, o se ponían a hablar y no, no daban esa apertura para participar, o sólo con algunos [estudiantes], que eran los de siempre (E10-F-UNAM).

Pues, yo tenía que preguntar, porque hay veces que se olvidaban de mí, y pues hay cosas que no entendía, de cosas que [los maestros] escribían en el pizarrón, pero ya al momento de preguntar me quedaba clara la duda, o pues, ya como que se aclaraba, sí (E12-M-UNAM).

Una estrategia para fomentar la participación es a través de los equipos de trabajo, pero aquí pueden surgir otras barreras que no dependen necesariamente del docente. $\mathrm{Al}$ respecto, la mayoría de nuestros entrevistados (siete alumnos) comentó no haber tenido muchas dificultades para ser incluidos en los equipos, no obstante, hubo casos en el que este proceso no fue tan sencillo, ya sea por la actitud de la propia persona con discapacidad o por la resistencia de los compañeros.

Pues, es que eso es lo que me cuesta un poco de trabajo todavía poderme integrar a los grupos de trabajo, como siento, o sea, todavía siento que en esa parte me llegan a hacer un poquito de rechazo (E16-F-UNAM). $[\ldots]$ siempre que hay trabajo en equipo yo me estreso muchísimo, o cuando tengo que exponer también es muy difícil, porque soy muy tímida y otra es porque a veces se quedan de ver en algún sitio y, hasta sí me esfuerzo por ir y todo, pero, es que no me dicen. Bueno, en parte también es mi culpa ¿no?, porque podría decirles ‘¿puedo ser parte de su equipo?' Pero no lo hago y siempre los profesores tienen que estar ahí, tengo que estarles diciendo que no tengo equipo y ya ahí me buscan un equipo (E17-F-UNAM).

Ser incluidos plenamente en las actividades áulicas, como al trabajo por equipo, tiene diferentes grados de complejidad, entre otras cosas por el desconocimiento que se tiene sobre las habilidades académicas de las personas con discapacidad y lo que pueden aportar. Pero la intervención oportuna de los profesores, como en el segundo testimonio, puede ser un facilitador para superar las reticencias de los compañeros.

El tercer aspecto es el de las actitudes del profesor. Como ocurre con cualquier estudiante, existen muchos factores que intervienen en el éxito (o fracaso) educativo, ya sean personales, económicos o sociales. Sin embargo, en los alumnos con discapacidad, las actitudes del personal que labora en las instituciones educativas tiene una gran influencia en su desarrollo académico (Wray, 2011).

En la UNAM, la mayoría de los entrevistados (siete) destacó la disposición de sus profesores para escucharlos, hacer ajustes a sus clases y apoyarlos: "No, aquí sí ninguno, ya a partir del CAH y de la carrera, no tuve problemas ni con profesores, ni con compañeros, y si las llegaba a tener eran diferencias, ya no digamos en el sentido de inclusión, sino más bien en un sentido ideológico, de ideas políticas o estas cuestiones" (E13-M-UNAM).

En otros casos, la situación no fue tan favorable: "Han sido menos los [maestros] que me han apoyado, mientras más avanzo en la Facultad, en las 
materias, es con menos frecuencia [...] Sí llevo justificante, pero, a pesar de eso, a muchos no les importa" (E18-M-UNAM).

Aquí, podrían estar interviniendo otras variables, que sería necesario explorar más a fondo, como los contenidos y perfiles de la carrera, la formación docente y la información previa sobre la presencia de estudiantes con discapacidad en sus clases.

Para otros alumnos, las experiencias fueron diversas, dependiendo de la personalidad y estilo de cada profesor: "No, o sea, cada clase es diferente, había profesores súper organizados, con los que jamás tuve ningún problema ni nada, pero había otros que pues no les podía sacar mucho a sus clases en realidad, pero igual te calificaban, entonces, en ese momento sí fue difícil" (E14-F-UNAM).

Finalmente, los estudiantes señalaron ciertos aspectos que podrían mejorarse, como el uso de distintas estrategias didácticas (tres alumnos), las formas de evaluación (dos) y la formación en inclusión por parte del personal de la institución (dos).

Se necesita que hubiera alguien, una persona especializada en la inclusión de personas con discapacidad a la educación, que pudiera orientar a los profesores para que supieran cómo evaluar y cómo tratar a la persona con discapacidad (E12-M-UNAM).

[...] hablando digamos de mi situación particular, hablando de la cuestión académica, pues sí pienso que debiese haber algún otro tipo de dispositivos de enseñanza que facilitaran el acceso a la explicación del profesor, porque por ejemplo en ese caso de las mate y la estadística sí fue dificultoso ¿no? O sea, como que ahí se notó más el tema de la discapacidad visual (E11-M-UNAM).

\section{Las barreras y facilitadores para la docencia en la UJAT}

Como hemos dicho, la diversificación de las formas de presentación beneficia no sólo a los estudiantes con discapacidad, sino a todos en general, porque permiten plantear diferentes vías para el aprendizaje y desarrollar las habilidades académicas, para la toma de decisiones y el trabajo colaborativo. Sobre este aspecto, los estudiantes de la UJAT coincidieron en que la clase expositiva era la estrategia más utilizada (cinco alumnos). Otras barreras fueron el uso continuo del pizarrón (cuatro), el trabajo con materiales impresos (cuatro) y la proyección de imágenes (cuatro).

Cabe señalar que, en esta Universidad, la muestra tuvo una mayor presencia de personas con discapacidad visual, seis de los nueve entrevistados, y también había una persona con discapacidad auditiva, de manera que la frecuencia registrada fue más alta; incluso, varios de los estudiantes señalaron más de una barrera: "acá los maestros lo ponen todo en el pizarrón y yo no lo puedo ver, entonces, me tienen que ayudar $[\ldots]$ no es por ser malas personas, sino que a veces yo no pregunto, no digo nada" (E9-F-UJAT).

Sobre los materiales impresos, uno de los entrevistados apuntó: "No me sirven mucho, porque los profesores entregan copias o libros que tienen letras muy pequeñas y yo no los puedo ver. [Entrevistador: ¿Y cómo le haces para solucionarlo?] Pues, generalmente me los leen o tengo que sacar copias más amplias" (E6-M-UJAT).

Otra estrategia utilizada por los docentes es la exposición de temáticas por equipos. Sobre esto, la mayoría reconoció no haber tenido dificultades para ser incluido (siete alumnos); algunos lo vieron como un proceso de adaptación entre ellos, sus maestros y sus compañeros, mientras que, para otros, las tensiones resultantes son producto de la complejidad del trabajo y de la comunicación, no necesariamente de su discapacidad.

Sí, me uno con mis compañeros, a veces ellos me buscan, a veces yo los busco a ellos, pero mayormente prefiero que me busquen a mí. Entonces, ya ellos me buscan y ya nos ponemos de acuerdo y todo. A veces sí, si las tareas son muy complejas, pues las tareas las hacemos en la casa de alguien, o aquí mismo 
en la escuela, por eso, tampoco tengo ningún problema (E8-F-UJAT).

Sin embargo, uno de los estudiantes señaló que, desde el inicio de la carrera, había enfrentado muchos problemas para incorporarse a los equipos: "Hay integrantes que no quieren trabajar conmigo y hay integrantes que quieren trabajar conmigo. Es que ellos sólo quieren estar con los mejores [...] Entonces, me quedo esperando, hasta que ellos se arrepienten solitos, si me necesitan o no me necesitan, y me doy cuenta que me quieren integrar" (E5-M-UJAT).

En este caso, el entrevistado indicó no haber recibido apoyo ni de los docentes, ni de sus compañeros. En contraste, varios de los entrevistados (cinco alumnos) comentaron que la mayoría de sus profesores los habían ayudado: "cuando hay que hacer algún trabajo en la clase, por equipo, o hay actividades culturales, o recreativas o de inclusión, los maestros y los chamacos siempre me invitan a participar, y a incluirme pues, dentro de las actividades para colaborar" (E1-M-UJAT).

En el tema de la evaluación, las opiniones fueron más diversas. Los estudiantes con discapacidad visual y auditiva enfrentaron mayores problemas, principalmente por las pocas opciones que hay para evaluarlos (cuatro) y el tiempo para presentar los trabajos (cuatro).

Tengo que conformarme con el examen oral y los maestros también porque no hay de otra, no sé si sería posible (E2-M-UJAT).

$[\ldots]$ hay maestros que sí me dan el mismo tiempo que a los otros y hay maestros que sí me esperan un poquito, porque me dicen: 'no, pues, no te voy a exigir que me lo traigas ahorita [el trabajo], porque necesitas un poquito más tiempo' $[\ldots]$, pero algunos no, algunos me dicen que de plano se lo tengo que entregar rápido, al igual que los demás (E7-M-UJAT).
Llama la atención que algunos profesores consideren que hacer los ajustes necesarios, en especial para los exámenes y los trabajos finales, les da a los estudiantes con discapacidad ciertas ventajas frente a sus compañeros. De manera que, en aras de la imparcialidad, intentan tratar a todos por igual, cuando en realidad los programas educativos tienen múltiples barreras porque están diseñados para alumnos 'promedio'.

No, lo hago [la evaluación] igual que mis compañeros, en eso no hay diferencia. Al menos, los maestros siempre han dicho que en el aula no hay preferencia porque yo esté en las condiciones en las que estoy y, pues eso es bueno, porque así no me siento preferente hacia mis compañeros (E4-F-ujat).

[...] siempre trato de entregarlos [los trabajos] cuando lo piden. No sé si me darían más tiempo, porque los maestros quieren, tratan a todos por igual (E8-F-UJAT).

El segundo componente de la docencia inclusiva es el de las formas de participación y compromiso. Aquí los entrevistados (ocho alumnos) sostuvieron que la mayoría de sus profesores trataban de incluirlos en las clases, ya sea a través de actividades individuales o grupales, siempre desde la perspectiva de la igualdad para todos los estudiantes.

[...] como todos, levanto mi mano, pido mi palabra, ya cuando el maestro decide, pues ya digo lo que tengo que decir y, en equipo, pues sí participo, hay cosas que sí, como le dije, cuando es hacer diapositivas lo único en que los apoyo es trayéndoles material [...] (E3-M-UJAT)

[...] siempre me apoyan [los maestros], me gusta participar, que es lo más importante que no me hago de menos y que no me subestimo a mí mismo, vaya, siempre me gusta, participar en cuestiones así (E1-M-UJAT). 
Sólo un estudiante señaló haber tenido algunas barreras para participar en clase: "No puedo participar, porque tengo un problema de nervio $[. .$.$] y eso$ es lo que me provoca, no puedo a como yo quiero decir mis palabras [...] es algo difícil. El maestro no ayuda, a veces se olvidan de mí, es difícil. Algunos sí ayudan, otros no mucho" (E5-M-UJAT).

A la par de las formas de presentación y de participación, está el tema de las actitudes del profesor. Aquí, igual que en la UNAM, las experiencias son muy variadas, poco más de la mitad de los estudiantes (cinco) expresó que, en general, los docentes eran atentos a sus necesidades y estaban dispuestos a escucharlos, aunque también reconocieron que en esto intervienen tanto la personalidad del maestro, como el proceso de negociación entre ellos.

Sí, pues generalmente así es, eh cuando llega el primer día de clases, el maestro llega y al final de las clases, le comento mi manera de trabajar, la discapacidad que tengo, los límites que tengo, cuánto me limita la discapacidad y, pues, ya llegamos a un acuerdo de trabajar, de utilizar ciertas herramientas, para poder aprender (E6-M-UJAT).

Sin embargo, otros enfrentaron algunas barreras (cuatro alumnos), ya sea por la falta de información previa sobre su presencia en las clases, la escasez de estrategias para incluirlos y las actitudes de los docentes.

Yo no sé si es que en mí se nota mucho la ceguera o no sé qué. Pero, la maestra el primer día que entró [al salón] dijo: 'Ay, ¿quién es ella?, ¿qué hago?' Dijo: 'es que a mí nadie me preparó, nadie me dijo' (E9-F-UJAT).

Son maestros, por lo regular, digamos son un poquito exigentes, son estrictos [...] pues quieren tratarme como un alumno normal, lo entiendo y lo agradezco, incluso. Pero, a veces, hay cosas que parece ser que no entienden $[\ldots]$ a veces piensan que en realidad quiero evadir alguna cuestión e interpretan eso como una cuestión de evasión o justificación, pero hay veces que, definitivamente por alguna razón no puedo (E2-M-UJAT).

Finalmente, los alumnos hicieron algunas recomendaciones, como la capacitación docente para la inclusión (tres estudiantes), que los profesores estuvieran atentos a sus necesidades (dos) y recibir un seguimiento más puntual de su trayectoria, por ejemplo, a través de las tutorías (uno).

algunos [maestros] sí necesitan, creo, que un poco más de capacitación para trabajar con nosotros. Pues, porque a veces, no sé, creo que no tienen la técnica o las herramientas para poder apoyarnos, no saben cómo, pues, trabajar esto (E6-M-UJAT).

Pues, yo necesito asesoría para poder realizar mi estudio adelante, que me pueda ayudar [en] los temas que se me van dificultando, un apoyo para resolver los problemas, como un guía (E5-M-UJAT).

\section{Análisis de resultados}

La inclusión de los estudiantes con discapacidad tiene diferentes implicaciones dependiendo del nivel al que estén referidos los cursos de acción. Particularmente, en nuestro país, gran parte de los esfuerzos en la educación superior se han hecho en lo académico-organizacional, es decir, en el ámbito de la normativa para las funciones sustantivas, el trabajo administrativo y los espacios institucionales (PérezCastro, 2018). En ello, el gobierno federal ha tenido una fuerte incidencia, ya que, como resultado de la expansión del enfoque de la inclusión, en las últimas décadas, se han emitido leyes, políticas y programas para ampliar las oportunidades educativas de este sector de la población. La respuesta de los establecimientos educativos ha sido diversa, pero, fundamentalmente, se ha centrado en las adaptaciones físicas, la implementación de algunos servicios especializados, la distribución de becas y la elaboración 
de lineamientos generales para la inclusión (Cruz y Casillas, 2017; Pérez-Castro, 2016).

A nivel de lo académico-pedagógico, es decir, de los planes de estudio, el desarrollo de las clases, los procesos de evaluación y acreditación, y la gestión de los cursos o seminarios, las medidas han sido más moderadas, no sólo por las implicaciones que esto tiene en las actividades de los profesores y estudiantes, sino también por las distintas barreras que rodean al trabajo áulico. En nuestra investigación, tanto en la UNAM como en la UJAT, la organización de las clases, principalmente en lo concerniente a las estrategias didácticas y las formas de presentación, fue una de las barreras más señaladas por los estudiantes. En este aspecto coinciden otras investigaciones, en donde se destacan las dificultades que las personas con discapacidad enfrentan cuando los docentes priorizan el formato de sesiones magistrales (Fuller, Bradley et al., 2004; Fuller, Healy et al., 2004). Asimismo, los resultados nos muestran que las barreras se incrementan en los alumnos con discapacidad visual y auditiva, lo que nos remite al argumento de que la discapacidad no está en los individuos, sino que es resultado de la interacción entre las condiciones del contexto y las capacidades de las personas.

En nuestra investigación, la evaluación fue otro rubro problemático para los estudiantes, las barreras más señaladas fueron la utilización de pruebas escritas o ensayos, el limitado repertorio de estrategias y los tiempos para contestar los exámenes o entregar los trabajos. No obstante, a la par, los entrevistados reconocieron el apoyo de sus profesores que, a través de los ajustes en los formatos y tiempos para la evaluación, hicieron que este proceso fuera más accesible. Aquí es importante subrayar dos cosas, por una parte, la importancia de la comunicación entre los docentes y alumnos. En ambas universidades, los estudiantes comentaron que cuando lo solicitaron, sus profesores los habían ayudado, es decir, los facilitadores no sólo dependen de estos últimos, sino también de las habilidades de las personas con discapacidad para plantear sus necesidades y co-desarrollar los ajustes y apoyos (Yuknis y Bernstein, 2017).

Por otra parte, hay que reconocer que los márgenes de acción de los docentes suelen ser acotados, porque tanto ellos como los alumnos tienen que ceñirse a lo que dispone el régimen académico (Baquero et al., 2009). Estas disposiciones no siempre pueden modificarse, aun en el nivel universitario en donde el principio de libertad de cátedra es altamente valorado. En ese sentido, es importante que las instituciones adecuen la normativa institucional, a partir del trabajo colegiado de directivos, profesores y estudiantes con discapacidad; no se trata de darles ventajas frente a sus compañeros, sino de reconocer las barreras que se pueden producir a partir de normas y condiciones que fueron hechas para estudiantes promedio.

En cuanto a las formas de participación y compromiso, la investigación nos permitió observar la estrecha relación que existe entre ellas y las formas de presentación en el contexto de las dos universidades aquí consideradas, algo que hasta ahora había sido explorado en instituciones de otros países (AHEAD, 2017; Healy et al., 2006; Konur, 2006). Entre las barreras mencionadas por los alumnos estuvieron las dificultades para participar en las sesiones y para mantener el contacto con sus compañeros y profesores; llama la atención que en ambas universidades, algunos estudiantes hayan advertido que, en ocasiones, estos últimos se olvidaban de su presencia en el aula. Lo anterior tendría que explorarse con más detenimiento, pero, inicialmente, podría estar apuntando hacia la necesidad de fortalecer las prácticas docentes para promover la participación, la colaboración y el diálogo entre todos los miembros de la clase.

En este aspecto, el trabajo por equipo fue el facilitador más señalado por los entrevistados, tanto en la UNAM como en la UJAT. Sin duda, ésta es una buena estrategia para abrir espacios a la participación (AHEAD, 2017; Fernández, 2013), aunque aquí pueden intervenir otros factores que no sólo dependen 
del profesor, como las actitudes de los alumnos sin discapacidad, así como las habilidades y disposición de las propias personas con discapacidad. En nuestra investigación, encontramos las dos situaciones, algunos entrevistados comentaron sobre las reticencias de sus compañeros para incluirlos, mientras que otros reconocieron sus propias limitaciones para trabajar en equipo.

Esto nos regresa al tema del trabajo colaborativo entre los profesores y los alumnos con discapacidad; en los primeros, se requiere fortalecer la formación para la docencia inclusiva, a fin de que puedan detectar este tipo de barreras y diseñar estrategias para superarlas, mientras que, en los segundos, es importante desarrollar sus habilidades para la toma de decisiones, la participación y la negociación con sus compañeros. En ambos casos, la intervención de las instituciones educativas es fundamental, a través de programas para la formación continua y el asesoramiento de los docentes, los servicios de apoyo a los estudiantes y el fortalecimiento de sus capacidades para que puedan incluirse plenamente en las clases y en la dinámica institucional (Fernández-de Álava et al., 2013).

Por último, está la cuestión de las actitudes hacia la inclusión. En las dos universidades de nuestra investigación, la mayoría de alumnos destacó la disposición de sus maestros para escucharlos y apoyarlos. De acuerdo con ellos, lo anterior depende mucho de la personalidad del profesor, lo que, si bien es un factor relevante, también apunta hacia la cultura institucional. Superar las barreras para la inclusión conlleva el desarrollo de una cultura, con estrategias descendentes y ascendentes (Lawrie et al., 2017), en donde todos se sientan reconocidos y tengan acceso a una educación de calidad y en equidad de condiciones.

\section{Consideraciones finales}

El propósito de este artículo fue acercarnos a tres componentes de la docencia inclusiva: las formas de presentación, las formas de participación y compromiso, y las actitudes hacia la inclusión, desde la perspectiva de los estudiantes con discapacidad. Al respecto, cabría decir que analizar la docencia en el nivel superior es una tarea compleja, en primer lugar, porque ésta es sólo una de las diferentes funciones que hacen los académicos y en segundo, por la diversidad de factores que intervienen en ella, ya sean disciplinarios, pedagógicos e institucionales. Adicionalmente, en México, la inclusión en educación superior es bastante reciente y, aunque se han implementado varias medidas, muchas se han quedado a nivel de los reglamentos, los servicios generales y la adaptación de los espacios físicos. Las condiciones para la docencia inclusiva han sido un aspecto poco atendido.

Los resultados de nuestra investigación muestran que los alumnos con discapacidad aún enfrentan múltiples barreras en los tres elementos del trabajo docente aquí considerados, lo que nos hace insistir en el compromiso de desarrollar políticas y estrategias que incluyan los diferentes factores que intervienen en él, como los contenidos curriculares, las condiciones institucionales y el perfil de los maestros y estudiantes, entre otros. Ciertamente, los profesores universitarios pueden contribuir implementando sus propios ajustes y apoyos; para ello, sin duda necesitan formarse en materia de inclusión y discapacidad, pero también en asuntos más concretos, como las barreras pedagógicas, las estrategias para superarlas, el trabajo colaborativo con sus colegas y con los estudiantes, y los servicios disponibles en la institución.

Asimismo, hay reconocer que la construcción de ambientes inclusivos no sólo depende de la voluntad de los docentes, sino también de las características del grupo. Las actitudes de los alumnos pueden facilitar u obstaculizar la inclusión de sus compañeros con discapacidad. A la par, es muy importante que estos últimos fortalezcan sus habilidades para plantear sus necesidades, identificar sus fortalezas, codiseñar los ajustes y establecer redes de apoyo con 
sus pares. En nuestra investigación, encontramos distintas situaciones que dan cuenta del peso que los docentes y alumnos con y sin discapacidad tienen en la relación barreras-facilitadores.

Finalmente, queremos señalar la importancia de construir una cultura de la inclusión, que se traduzca en acciones concertadas entre directivos, profesores y estudiantes. La inclusión educativa es un derecho

\section{Referencias}

Abdó, Jorge (2000), Compromisos con la sociedad, Villahermosa, UJAT.

AHEAD (2017), Universal design for learning. A best practice guideline, Dublin, Association for Higher Education Access \& Disability.

Ainscow, Mel (1995), Necesidades especiales en el aula. Guía para la formación del profesorado, Madrid, Narcea.

Ainscow, Mel, John Beresford, Alma Harris, David Hopkins y Mel West (2001), Crear condiciones para la mejora del trabajo en el aula. Manual para la formación del profesorado, Madrid, Narcea.

Aquino, Katherine, Taghreed Alhaddab y Eunyoung Kim (2017), 'Does disability matter? Students' satisfaction with college experiences", en Eunyoung Kim y Katherine Aquino (eds.), Disability as diversity in higher education, Nueva York, Routledge, pp. 47-60.

Baquero, Ricardo, Flavia Terigi, Ana Toscano, Bárbara Brisciol y Santiago Sburlatti (2009), "Variaciones del régimen académico en escuelas medias con población vulnerable. Un estudio de casos en el área metropolitana de Buenos Aires", Revista Iberoamericana sobre Calidad, Eficacia y Cambio en Educación, vol. 7, núm. 4, pp. 293319, <https://revistas.uam.es/index.php/reice/ article/ view/5394/5833> [C onsulta: mayo de 2019].

Bolívar, Antonio (2012), "Justicia social y equidad escolar. Una revisión actual", Revista Internacional de Educación para la Justicia Social, vol. 1, núm. 1, pp. 9-45. <https:// que no puede dejarse únicamente a la voluntad de las personas, sino que debe ser una política transversal que abarque las diferentes dimensiones de la dinámica institucional. En esta tarea deben participar todos los miembros de la comunidad universitaria, apoyándose de otras instancias públicas y privadas que han trabajado por la inclusión de las personas con discapacidad. revistas.uam.es/riejs/article/view/308/310> [Consulta: junio de 2019].

Brandt, Synnove (2011), "From policy to practice in higher education: the experiences of disabled students in Norway", International fournal of Disability, Development and Education, vol. 58, núm. 2, pp. 107-120. DOI: https://www.tandfonline.com/doi/full/10.1080/1034 912X.2011.570494 [Consulta: julio de 2019].

Brogna, Patricia y Damián Rosales (2013), Diagnóstico sobre el estado que guarda la atención a la temática de la discapacidad en la UNAM desde la perspectiva de los derechos humanos, México, UNAM-PUDH.

Cruz, Rodolfoy MiguelCasillas(2017), "Lasinstituciones de educación superior y los estudiantes con discapacidad en México", Revista de la Educación Superior, vol. 46, núm. 181, pp. 37-53.

Defensoría de los Derechos Universitarios (DDU) (2019), "Defensoría de los Derechos Universitarios", México, UNAM, <https://www.defensoria.unam. $\mathrm{mx} />$ [Consulta: julio de 2019].

Delgado-Sanoja, Hellman y Gisela Blanco-Gómez (2016), "Inclusión en la educación universitaria. Las palabras y experiencias detrás del proceso", Revista Electrónica Educare, vol. 20, núm. 2, pp. 1-18, DOI: 10.15359/ ree.20-2.9 [Consulta julio de 2019].

Echeita, Gerardo y Mel Ainscow (2011), "La educación inclusiva como derecho. Marco de referencia y pautas de 
acción para el desarrollo de una revolución pendiente", Tejuelo, núm. 12, pp. 26-46, <https://mascvuex.unex. es/revistas/index.php/tejuelo/article/view/2497> [Consulta: mayo de 2019.

Fernández, José María (2013), “Competencias docentes y educación inclusiva", REDIE. Revista electrónica de investigación educativa, vol. 2, núm. 15, pp. 82-99, <https:/ / redie.uabc. $\mathrm{mx} / \mathrm{redie} / \mathrm{article} / \mathrm{view} / 445 / 610>$ [Consulta: junio de 2019]

Fernández-de-Álava, Miren, Aleix Barrera-Corominas y Anna Díaz-Vicario (2013), "La inclusión en instituciones iberoamericanas de educación superior. Buenas prácticas para el acceso y la permanencia de estudiantes con discapacidad", Revista Iberoamericana de Educación, núm. 63, pp. 99-113.

Florian, Lani y Holly Linklater (2010), "Preparing teachers for inclusive education: using inclusive pedagogy to enhance teaching and learning for all", Cambridge Fournal of Education, vol. 40, núm. 4, pp. 369-386, <http://www.mofet.macam.ac.il/amitim/ iun/Documents/Florian_and_Linklater_preparing_ teachers_for_inclusive_education.pdf $>$ [Consulta: junio de 2019].

Florian, Lani y Kristine Black-Hawkins (2011), "Exploring inclusive pedagogy", British educational research journal, vol. 37, núm. 5, pp. 813-828, DOI: https://onlinelibrary. wiley.com/doi/abs/10.1080/01411926.2010.501096 [Consulta 28 de mayo de 2019].

French, Swain (2017), Disabled people and employment. A study of the working lives of the visually impaired physiotherapists, Londres, Routledge.

Fuller, Mary, Andrew Bradley y Mick Healy (2004), "Incorporating disabled students within an inclusive higher education environment", Disability \& Society, vol. 19, núm. 5, pp. 455-468, <https://www.tandfonline. com/doi/abs/10.1080/0968759042000235307> [Consulta: abril de 2018].

Fuller, Mary, Mick Healy, Andrew Bradley y Tim Hall (2004), "Barriers to learning: a systematic study of the experience of disabled students in one university", Studies in Higher Education, vol. 29, núm. 3, pp. 303-318,
DOI: https://www.tandfonline.com/doi/abs/10.1080 /03075070410001682592 [Consulta: abril de 2018].

Gairín, Joaquín y Cecilia Suárez (2014), "Clarificar e identificar los grupos vulnerables”, en Joaquín Gairín (coord.), Colectivos vulnerables en la Universidad. Reflexiones y propuestas para la intervención, Madrid, Wolters Kluwer, pp. 33-61.

Gil, Candita (2006), Plan estratégico de desarrollo 2006-2016, Villahermosa, UJAT.

Healy, Mick, Andrew Bradley, Mary Fuller y Tim Hall (2006), "Listening to students. The experience of disabled students of learning at university", en Mike Adams y Sally Brown (eds.), Towards inclusive learning in higher education, Londres, Routledge, pp. 32-43.

Hénard, Fabrice y Deborah Roseveare (2012), Fostering quality teaching in higher education: policies and practices, París, OECD.

Hewett, Rachel, Graeme Douglas, Michael McLinden y Sue Keil (2017), "Developing an inclusive learning environment for students with visual impairment in higher education: progressive mutual accommodation and learner experiences in the United Kingdom", European Journal of Special Needs Education, vol. 32, núm. 1, pp. 89-109, DOI: https://www.tandfonline.com/ doi/abs/10.1080/08856257.2016.1254971 [Consulta: febrero de 2019].

Hopkins, Laurence (2011), "The path of least resistance: a voice-relational analysis of disabled students' experiences of discrimination in English universities", International Fournal of Inclusive Education, vol. 15, núm, 7, pp. 711-727, DOI:https://www.tandfonline.com/doi/abs/10.1080/ 13603110903317684 [Consulta: febrero de 2019].

Imbernón, Francisco (2000), "Un nuevo profesorado para una nueva universidad. ¿Conciencia o presión?”, Revista Interuniversitaria de Formación del Profesorado, núm. 38, pp. 37-46, <http://redined.mecd.gob.es/xmlui/ bitstream/handle/11162/30960/014200030743. pdf ? sequence $=1 \&$ is Allowed $=y>$ C Consulta: septiembre de 2018].

Jackson, Philip (2001), La vida en las aulas [6 $6^{\mathrm{a}}$ ed.], Madrid, Morata, pp. 11-26. 
Konur, Ozcan (2006), "Teaching disabled students in higher education", Teaching in higher education, vol. 11, núm. 3, pp. 351-363, DOI: https://tandfonline.com/ doi/abs/10.1080/13562510600680871?src=recsys\&j ournalCode $=$ cthe20 [Consulta: enero de 2018].

Lawrie, Gwen, Elizabeth Marquis, Eddie Fuller, Tara Newman, Mei Qiu, Milton Nomikoudis, Frits Roelofs y Lianne van Dam (2017), "Moving towards inclusive learning and teaching: A synthesis of recent literature", Teaching \&̊ learning inquiry, vol. 5, núm. 1, pp. 1-13, $<$ https:/ / files.eric.ed.gov/fulltext/EJ1148444.pdf> [Consulta: junio de 2019].

Lombardi, Allison y Adam Lalor (2017), "Faculty and administrator knowledge and attitudes regarding disability", en Eunyoung Kim y Katherine Aquino (eds.), Disability as diversity in higher education, Nueva York, Routledge, pp. 107-121.

López, Alicia (2015), "El Comité de Atención a las Personas con Discapacidad en la Universidad Nacional Autónoma de México", en Norma del Río (comp.), Políticas inclusivas en la educación superior de la Ciudad de México, México, CDHDF, pp. 79-89.

McKay, Loraine (2016), "Beginning teachers and inclusive education: frustrations, dilemmas and growth", International fournal of Inclusive Education, vol. 20, núm. 4, pp. 383-396, DOI: https://www.tandfonline. com/doi/abs/10.1080/13603116.2015.1081635?jour nal Code=tied20 [Consulta: junio de 2018].

Moreno, María (2005), “Integración/inclusión de las personas con discapacidad en la educación superior", en Informe sobre la educación superior en América Latina, Caracas, UNESCO-IESALC, pp. 144-155.

Moriña, Anabel y Rafael Carballo (2018), "Profesorado universitario y educación inclusiva: respondiendo a sus necesidades de formación", Psicología Escolar y Educacional, número especial, pp. 87-95. <http:// www.scielo.br/pdf/pee/v22nspe/2175-3539-pee-22spe-87.pdf> [Consulta: enero de 2019].

Norwich, Brahm (2008), Dilemmas of difference, inclusion and disability, Londres, Routledge.

OECD (2003), Disability in higher education, París, OECD.
OMS (2011), Informe mundial sobre la discapacidad, Ginebra, OMS-BM.

Palmeros, Guadalupe (2014), "Prácticas y experiencias en la División Académica de Educación y Artes de la Universidad Juárez Autónoma de Tabasco”, en Joaquín Gairín, Guadalupe Palmeros y Adoración Barrales, Universidad y colectivos vulnerables, México, Ediciones del Lirio, pp. 131-139.

Parrilla, Ángeles (2002), "Acerca del origen y sentido de la educación inclusiva", Revista de Educación, núm. 237, pp. 11-29.

Pérez-Castro, Judith (2018), "Condiciones para la inclusión de los estudiantes universitarios con discapacidad en el trabajo áulico", Memorias del Congreso internacional de investigación Academia fournals, vol. 10, núm. 3, pp. 2615-2621

Pérez-Castro, Judith (2016), "La inclusión de las personas con discapacidad en la educación superior en México", Sinéctica. Revista Electrónica de Educación, núm. 46, pp. 1-15. <https://sinectica.iteso.mx/index.php/SINEGTICA/ article/ view/614> [Consulta: mayo de 2018].

Piña, José (2016), 1er. Informe de actividades 2016, Villahermosa, UJAT.

Piña, José (2012), Plan de desarrollo institucional 2012-2016, Villahermosa, UJAT.

Red2Red (2013), Discapacidad, estudios superiores y mercado de trabajo. Barreras de acceso y repercusión en la inserción laboral, Madrid, Red2Red Consultores.

Ríos, Telma, María Celis, Miguel Robles y Marco Bonaparte (2013), "Unidad de atención para personas con discapacidad", Revista Digital Universitaria, vol. 14, núm. 12, pp. 1-11 <http://www.revista.unam.mx/vol.14/num12/ art54/art54.pdf> [Consulta: abril de 2017].

Rodríguez, Gregorio, Javier Gil y Eduardo García (1996), Metodología de la investigación cualitativa, Granada, Aljibe.

Stake, Robert (2003), "Case studies", en Norman K. Denzin e Yvonna S. Lincoln (eds.), Strategies of qualitative inquiry [2 ${ }^{\mathrm{a}}$ edition], Thousand Oaks, Sage, pp. 134-164.

UNAM-DGACO (2019), Guía de apoyos y servicios de atención para personas con discapacidad en la UNAM, México, UNAM, <https://unapdi.unam.mx/> [Consulta: marzo de 2019]. 
Vickerman, Philip y Milly Blundell (2010), "Hearing the voices of disabled students in higher education", Disability Eீ Society, vol. 25, núm. 1, pp. 2132, DOI:https://www.tandfonline.com/doi/ abs/10.1080/09687590903363290 [Consulta: febrero de 2018].

Waterfield, Judith, Bob West y Melanie Parker (2006), "Supporting inclusive practice. Developing an assessment toolkit", en Mike Adams y Sally Brown (eds.), Towards inclusive learning in higher education, Londres, Routledge, pp. 79-94.

Wilches-Chaux, Gustavo (1993), "La vulnerabilidad global”, en A. Maskrey (comp.), Los desastres no son naturales, San José de Costa Rica, Red de Estudios Sociales en Prevención de Desastres en América Latina. Wilson, Kristi, Ellizabeth Getzel y Tracey Brown (2000), "Enhancing the post-secondary campus climate for students with disabilities", fournal of Vocational
Rehabilitation, núm. 14, pp. 37-50, <https:// thinkcollege.net/sites/default/files/files/resources/ Wilson_enhancing.pdf> [Consulta: enero de 2017].

Wray, Mike (2011), Disabled learners and barriers to higher education, Warwick, Coventry and Warwickshire Aimhigher.

Yuknis, Christina y Eric Bernstein (2017), "Supporting students with non-disclosed disabilities: A collective and humanizing approach", en Eunyoung Kim y Katherine Aquino (eds.), Disability as diversity in higher education. Policies and practices to enhance student success, Nueva York, Routledge, pp. 3-18.

Zabalza, Miguel (2009), "Ser profesor universitario hoy", La cuestión universitaria, núm. 5, pp. 68-80, <http:// polired.upm.es/index.php/lacuestionuniversitaria/ article/view/3338> [Consulta: noviembre de 2018].

Zabalza, Miguel y María Zabalza (2012), Profesores y profesión docente. Entre el «sen» y el «estar», Madrid, Narcea.

Cómo citar este artículo:

Pérez-Castro, Judith (2021), "Condiciones para la docencia inclusiva: análisis desde las barreras y los facilitadores", Revista Iberoamericana de Educación Superior (RIES), vol. XII, núm. 33, pp. 138-157, DoI: https://doi.org/10.22201/iisue.20072872e.2021.33.862 [Consulta: fecha de última consulta]. 